\title{
The value of complete blood count for the prognosis analysis of preoperative esophageal squamous cell carcinoma
}

\author{
Xiang $\mathrm{Lv}^{1 \dagger}$, Songtao $\mathrm{Han}^{2 \dagger}$, Bin $\mathrm{Xu}^{1 \dagger}$, Yuqin Deng ${ }^{3}$ and Yangchun Feng ${ }^{1 *}$
}

\begin{abstract}
Objective: To investigate the predictive value of preoperative complete blood count for the survival of patients with esophageal squamous cell carcinoma.

Methods: A total of 1587 patients with pathologically confirmed esophageal squamous cell carcinoma who underwent esophagectomy in the Cancer Hospital Affiliated to Xinjiang Medical University from January 2010 to December 2019 were collected by retrospective study. A total of 359 patients were as the validation cohort from January 2015 to December 2016, and the remaining 1228 patients were as the training cohort. The relevant clinical data were collected by the medical record system, and the patients were followed up by the hospital medical record follow-up system. The follow-up outcome was patient death. The survival time of all patients was obtained. The Cox proportional hazards regression model and nomogram were established to predict the survival prognosis of esophageal squamous cell carcinoma by the index, their cut-off values obtained the training cohort by the ROC curve. The Kaplan-Meier survival curve was established to express the overall survival rate. The 3-year and 5-year calibration curves and C-index were used to determine the accuracy and discrimination of the prognostic model. The decision curve analysis was used to predict the potential of clinical application. Finally, the validation cohort was used to verify the results of the training cohort.
\end{abstract}

Results: The cut-off values of NLR, NMR, LMR, RDW and PDW in complete blood count of the training cohort were $3.29,12.77,2.95,15.05$ and $13.65 \%$, respectively. All indicators were divided into high and low groups according to cut-off values. Univariate Cox regression analysis model showed that age $(\geq 60)$, NLR $(\geq 3.29)$, LMR $(<2.95)$, RDW $(\geq 15.05 \%)$ and PDW $(\geq 13.65 \%)$ were risk factors for the prognosis of esophageal squamous cell carcinoma; multivariate Cox regression analysis model showed that age $(\geq 60)$, NLR $(\geq 3.29)$ and LMR $(<2.95)$ were independent risk factors for esophageal squamous cell carcinoma. Kaplan-Meier curve indicated that age $<60, N L R<3.52$ and $L M R \geq 2.95$ groups had higher overall survival $(p<0.05)$. The 3 -year calibration curve indicated that its predictive probability overestimate the actual probability. 5 -year calibration curve indicated that its predictive probability was consistent with the actual probability. 5 c-index was 0.730 and 0.737 , respectively, indicating that the prognostic model had high accuracy and discrimination. The decision curve analysis indicated good potential for clinical application. The validation cohort also proved the validity of the prognostic model.

\footnotetext{
*Correspondence: paopao1987123@163.com

${ }^{+}$Xiang Lv, Songtao Han and Bin Xu contributed equally to this work.

${ }^{1}$ Clinical Laboratory Center, Cancer Hospital Affiliated to Xinjiang Medical University, Xinjiang, China

Full list of author information is available at the end of the article
}

(c) The Author(s). 2021 Open Access This article is licensed under a Creative Commons Attribution 4.0 International License, which permits use, sharing, adaptation, distribution and reproduction in any medium or format, as long as you give appropriate credit to the original author(s) and the source, provide a link to the Creative Commons licence, and indicate if changes were made. The images or other third party material in this article are included in the article's Creative Commons licence, unless indicated otherwise in a credit line to the material. If material is not included in the article's Creative Commons licence and your intended use is not permitted by statutory regulation or exceeds the permitted use, you will need to obtain permission directly from the copyright holder. To view a copy of this licence, visit http://creativecommons.org/licenses/by/4.0/ The Creative Commons Public Domain Dedication waiver (http://creativecommons.org/publicdomain/zero/1.0/) applies to the data made available in this article, unless otherwise stated in a credit line to the data. 
Conclusion: NLR and LMR results in complete blood count results can be used to predict the survival prognosis of patients with preoperative esophageal squamous cell carcinoma.

\section{Background}

Malignancy is a major public health problem in the world, according to the 2019 global cancer data report, esophageal cancer incidence and mortality rank the 7th and 6th in all cancer, respectively [1]. Esophageal cancer could be divided into two categories according to histological types, including esophageal squamous cell carcinoma (ESCC) and esophageal adenocarcinoma (EAC). Esophageal squamous cell carcinoma mainly distributed in Asia, accounting for about 90\%; esophageal adenocarcinoma mainly distributed in Europe, accounting for about $70-80 \%[2,3]$. The middle-aged and elderly had high incidence of esophageal cancer, the early clinical manifestations are mainly dysphagia, burning sensation under the sword, weight loss and other atypical symptoms, the later could be manifested as progressive dysphagia, so the early symptoms are easy to miss the diagnosis, most of the diagnosis is advanced, the fiveyear survival rate is only $10-20 \%[4,5]$, early and midterm esophageal cancer should be treated by surgery, but the overall survival rate has not changed significantly, so how to evaluate the prognosis of patients before surgery has important clinical significance for clinicians and patients, which could help clinicians to change the treatment plan, while improving the survival confidence of patients.

Over the past few decades, it has been confirmed that depth of invasion, lymph node metastasis, TNM stage, and various other factors [6] were important prognosis factors of esophageal cancer, meanwhile, more and more experiments in recent years have demonstrated that inflammation is associated with the survival of malignant tumors. Complete blood count $(\mathrm{CBC})$ is one of the most common clinical laboratory tests, and absolute counts of neutrophils, lymphocytes, and monocytes reflect the inflammatory response and the overall immune status of the body. Peripheral blood prognostic inflammatory markers include neutrophil to lymphocyte ratio (NLR), neutrophil to monocyte ratio (NMR), lymphocyte to monocyte ratio (LMR), red blood cell distribution width (RDW) and blood cell distribution width (PDW), which have been demonstrated to be closely related to the prognosis of various cancers $[6,7]$. In clinical laboratories, because blood routine tests are mandatory for patients and cheap, experimental data are relatively easy to obtain. Therefore, it is of great clinical significance to predict the prognosis of patients with esophageal squamous cell carcinoma by the results of blood routine test items.

\section{Materials and methods \\ Patient}

This retrospective study was approved by the Ethics Committee of Cancer Hospital Affiliated to Xinjiang Medical University. All cases of esophageal cancer were diagnosed by pathological results. A total of 1587 cases of esophageal squamous cell carcinoma were collected, who underwent esophagectomy and were diagnosed in the Cancer Hospital Affiliated to Xinjiang Medical University from January 2010 to December 2019. In them, 359 cases from January 2015 to December 2016 were selected as the validation cohort, and the remaining 1228 cases were as the training cohort. All patients with esophageal squamous cell carcinoma were pathologically staged by experienced oncologists according to the 8th edition of TNM staging issued by the American Joint Committee on Cancer (AJCC). Inclusion criteria:1) histopathologically confirmed ESCC without distant metastasis (TNM-I-III) from January 2010 to December 2019 were included in the study; 2) at least 6 lymph nodes were examined for pathological diagnosis; 3) no chemotherapy and/or radiotherapy before and after surgery; 4) preoperative $\mathrm{CBC}$ was obtained 1 time before esophagectomy. Exclusion criteria: 1) previous or concomitant other cancer; 2) serious complications or death within 30 days after surgery; 3 ) preoperative systemic inflammatory response syndrome (SIRS) or evidence of infection, autoimmune disease. The last follow-up date was 21 February 2021.

\section{Statistical analysis}

The area under the curve (AUC) was calculated by receiver operating characteristic curve (ROC) and the optimal cutoff value for continuous variables was calculated. The correlation between each indicator and pathological parameters was indicated by spearman correlation coefficient, univariate and multivariate analyses were performed using the Cox regression analysis model to assess the effect of multiple covariates on survival outcomes; nomograms were generated with Cox regression coefficients, Kaplan-Meier survival curves were established to express overall survival, their accuracy and discrimination were indicated using the 3- and 5-year calibration curves and c-index, their potential for clinical application was indicated using the decision curve analyses, and finally validated using the validation cohort. For all analyses, $p<0.05$ was defined as significant. Statistical analysis was performed using SPSS version 26.0 
(SPSS, Chicago, IL) and the rms, survival, survivalROC, and rmda packages of $\mathrm{R}$ language.

\section{Results}

Cut-off value of every test index

Taking whether the patient died in the final result as the outcome event, ROC curve was used to find out whether there was an appropriate cut-off value for every test items to predict the patient's death outcome, and then the significance of every indicators in prognosis was demonstrated. The cut-off values of relevant quantitative NLR, NMR, LMR, RDW, PDW and other indicators were $3.29,12.77,2.95,15.05$ and $13.65 \%$,respectively (Table 1, Fig. 1).

\section{Clinicopathological features of patients}

The clinicopathological characteristics of the patients in the training cohort $(n=1228)$ and validation cohort $(n=359)$ in Table 2. The male to female ratio was 2.21:1 and 2.55:1 in the training and validation cohorts, respectively. In the training cohort Age $>60(66.2 \%)$ and tumor location in the middle $(72.6 \%)$ was significantly higher than $<60(33.8 \%)$ and tumor location in the upper and lower segments (27.4\%). In the pathological stage, T3 (64.6\%) was the most depth of tumor invasion group, which was related to the diagnosis of advanced ESCC patients. $40.9 \%$ of ESCC patients had no lymph node metastasis. In Pathological staging, stage II (41.2\%) and III (50.8\%) patients accounted for the vast majority.

Based on the correlation between various indicators in the training cohort and pathological parameters (Table 3), It could be concluded that NLR, NMR and PDW were significantly correlated with Depth of invasion, Lymph node metastasis and Stage $(p<0.05)$, LMR is correlated with Lymph node metastasis, Differentiation and Stage $(\mathrm{p}<0.05)$, and RDW is correlated with Stage $(\mathrm{p}<0.05)$.

\section{Univariate and multivariate cox regression analysis of various indicators}

Univariate Cox regression analysis was used to calculate the value of gender, age, every classification index, tumor location, depth of invasion, lymph node metastasis,

Table 1 ROC Analysis Results of Significant Quantitative

\begin{tabular}{llll}
\hline Index & Cut-off value & AUC & P \\
\hline NLR & 3.29 & 0.615 & $<0.001$ \\
NMR & 12.77 & 0.516 & $<0.001$ \\
LMR & 2.95 & 0.598 & $<0.001$ \\
PDW & 15.05 & 0.489 & 0.275 \\
RDW & 13.65 & 0.498 & 0.179 \\
\hline
\end{tabular}

NOTE:NLR:neutrophil to lymphocyte ratio;NMR:neutrophil to monocyte ratio;LMR:lymphocyte to monocyte ratio;RDW: red blood cell distribution width;PDW: blood cell distribution width differentiation and pathological stage for the prognosis of esophageal cancer, respectively, and it could be found (Table 4$)$ that age $(p<0.001)$, NLR $(p<0.001)$, LMR $(\mathrm{p}<0.001)$, RDW $(p=0.049)$, PDW $(p=0.016)$, depth of invasion $(p<0.001)$, lymph node metastasis $(\mathrm{p}<0.001)$ and stage $(\mathrm{p}<0.001)$ were prognostic factors for esophageal cancer; multivariate Cox regression analysis could reveal that age $(\mathrm{p}<0.001)$, NLR $(\mathrm{p}<0.001)$, LMR $(p=0.001)$, depth of invasion $(\mathrm{p}<0.001)$, lymph node metastasis $(p=0.016)$ and stage $(p<0.036)$ were independent risk factors for the prognosis of esophageal cancer. The age $\geq 60$ years group showed a 1.341 -fold $(1.169-1.538)$ increased risk of death than the $<60$ years group;NLR $\geq 3.52$ group vs. $<3.52$ group, with a 1.578 fold (1.373-1.814) increased risk of death. The LMR < 2.95 group was associated with a 1.265 -fold (1.1071.445) increased risk of death compared with the LMR $\geq$ 2.95 group.

\section{Survival analysis results of significance detection indicators}

Based on the Cox regression multivariate analysis, we selected Age, NLR, and LMR to establish the survival curve. From the (Fig. 2A-C), it showed that the age $<60$, NLR $<3.52$ and LMR $\geq 2.95$ groups had higher overall survival.

\section{Nomogram development and internal validation}

Based on Cox regression multivariate analysis of the training cohort; the nomogram for predicting overall survival at 3 and 5 years in the training cohort was established by age, NLR, LMR, depth of invasion, lymph node metastasis, and stage (Fig. 3). The 3-year survival probability calibration plot indicated that the nomogram predicted risk overestimate the actual risk,the 5-year survival probability calibration plot indicated good accuracy between nomogram prediction and actual observation. Their C-index scores were 0.730 and 0.737 , indicating their good discrimination. The decision curve showed that it had good potential for clinical application (Fig. 4A-F).

At the end of the internal validation, the overall survival of every patient in the validation cohort was evaluated by a nomogram designed based on the training cohort. The calibration curves show good agreement in the 5-year survival probability between actual observed and nomogram predictions, and their 3-year actual observed risk was still overestimated. In the validation cohort, the 3- and 5-year C-index was 0.733 and 0.745 . These results indicate that the Nobel plot is a more accurate and useful tool for predicting overall survival in patients with operable ESCC (Fig. 5A-F). 

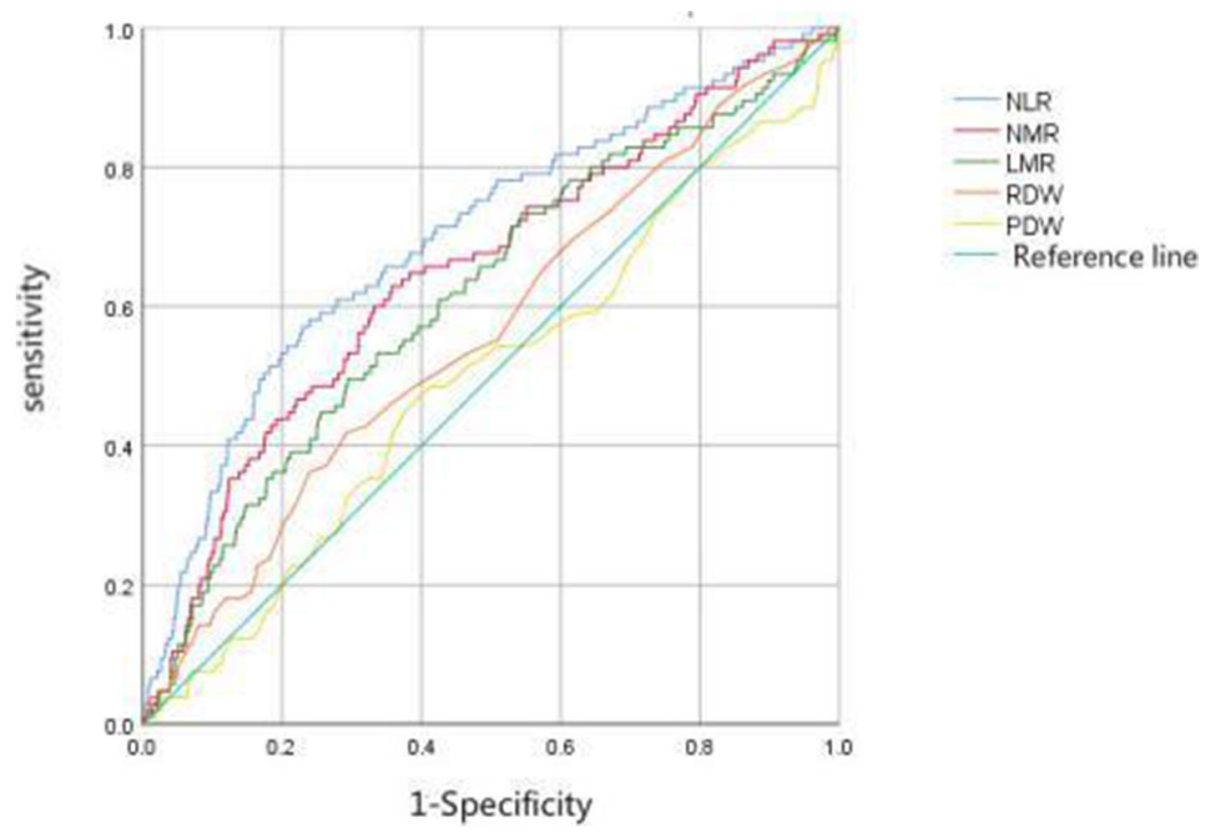

monocyte ratio;RDW: red blood cell distribution width;PDW: blood cell distribution width

\section{Discussion}

The incidence of esophageal cancer is high in the world. About 580,000 new cases were diagnosed worldwide, meanwhile, about 510,000 people died of esophageal cancer every year. In China, nearly 300,000 patients with esophageal cancer died in every year, accounting for more than $50 \%$ of the global deaths of esophageal cancer [8]. Now it is generally recognized that inflammation plays an increasingly important role in the development of tumors, and its mechanism may be include the inflammation releases cytokines and up-regulation of transcription factors, then leading to the generation and accumulation of a large number of oxygen free radicals, which could cause DNA damage and breakage in parenchymal cells, including stem cells, overexpression of proto-oncogenes, loss of function of tumor suppressor genes and up-regulation of genes that promote the cell cycle, leading to abnormal cell proliferation, thereby interfering with the stability of the body's microenvironment, accelerating tumor growth, invasion, metastasis and other processes, affecting the prognosis of tumors $[9,10]$. Many reports have shown that LMR, NLR and NMR, PDW, RDW as inflammatory indicators, were closely related to the prognosis of a variety of diseases and could be used as prognostic factors in a variety of malignant tumors $[7,11,12]$. In recent years, nomograms have been constructed for many cancers, and some of them were more reliable than traditional staging prediction [13]. Although the nomograms have many advantages, there were few nomograms to construct for
ESCC by CBC. We established nomograms by CBC indicators and pathological parameters to predict the prognosis of ESCC and validate its model performance.

In this study, we investigated the effect of inflammatory indicators in blood routine on the prognosis of preoperative esophageal squamous cell carcinoma. Based on univariate Cox regression analysis of training cohort, NLR $\geq$ 3.29, $\mathrm{LMR}<2.95, \mathrm{RDW} \geq 15.05 \%$ and $\mathrm{PDW} \geq 13.65 \%$ were risk factors for preoperative esophageal squamous cell carcinoma prognosis; multivariate Cox regression analysis model showed that NLR $\geq 3.29$ and $\mathrm{LMR}<2.95$ were independent risk factors for preoperative esophageal squamous cell carcinoma, which were the same as most studies; the HR of NLR $\geq 3.29$ was 1.578 (1.373-1.814; $P<0.001)$ than NLR $<3.29$, which was consistent with Arigami T [14] and Gao GD [15] studies, and NLR > 3.0 and NLR > 2.83 were effective predictors for preoperative esophageal carcinoma; the HR of $\mathrm{LMR}<2.95$ was 1.265 (1.107-1.445; $\mathrm{P}<0.001)$ than $\mathrm{LMR} \geq 2.95$, Huang $\mathrm{Y}$ [16] et al. believed that preoperative $\mathrm{LMR}<2.93$ predicted poor prognosis of patients with esophageal squamous cell carcinoma. Hu G [17] et al. did a meta-analysis and also concluded that low LMR could predict poor prognosis of esophageal cancer, meanwhile, Li KJ [18] believed that LMR and NLR could be used to predict the survival rate of patients with advanced esophageal cancer who also received chemoradiotherapy, and confirmed that LMR and NLR could predict the survival rate of patients with preoperative esophageal cancer in this study. Among other related blood routine test indicators, PDW had distinct 
Table 2 Clinicopathological features and CBC index of esophageal squamous cell carcinoma

\begin{tabular}{|c|c|c|c|c|}
\hline \multirow[t]{2}{*}{ characteristic } & \multicolumn{2}{|c|}{ Training cohort $(n=1228)$} & \multicolumn{2}{|c|}{ Validation cohort $(n=359)$} \\
\hline & Number of patients & Composition ratio (\%) & Number of patients & $\overline{\text { Composition ratio (\%) }}$ \\
\hline \multicolumn{5}{|l|}{ Sex } \\
\hline Male & 845 & $68.8 \%$ & 258 & $71.9 \%$ \\
\hline Female & 383 & $31.2 \%$ & 101 & $28.1 \%$ \\
\hline \multicolumn{5}{|l|}{ Age } \\
\hline$<60$ years & 415 & $33.8 \%$ & 111 & $30.9 \%$ \\
\hline$\geq 60$ years & 813 & $66.2 \%$ & 248 & $69.1 \%$ \\
\hline \multicolumn{5}{|l|}{ NLR } \\
\hline$<3.29$ & 864 & $70.4 \%$ & 252 & $70.2 \% \%$ \\
\hline$\geq 3.29$ & 364 & $29.6 \%$ & 107 & $29.8 \% \%$ \\
\hline \multicolumn{5}{|l|}{ NMR } \\
\hline$<12.77$ & 1013 & $82.5 \%$ & 289 & $80.5 \%$ \\
\hline$\geq 12.77$ & 215 & $17.5 \%$ & 70 & $19.5 \%$ \\
\hline \multicolumn{5}{|l|}{ LMR } \\
\hline$<2.95$ & 421 & $34.3 \%$ & 126 & $35.1 \%$ \\
\hline$\geq 2.95$ & 807 & $65.7 \%$ & 233 & $64.9 \%$ \\
\hline \multicolumn{5}{|l|}{ RDW } \\
\hline$<15.05 \%$ & 786 & $64 \%$ & 234 & $65.2 \%$ \\
\hline$\geq 15.05 \%$ & 442 & $36 \%$ & 125 & $34.8 \%$ \\
\hline \multicolumn{5}{|l|}{ PDW } \\
\hline$<13.65 \%$ & 1103 & $89.8 \%$ & 324 & $90.3 \%$ \\
\hline$\geq 13.65 \%$ & 125 & $10.2 \%$ & 35 & $9.7 \%$ \\
\hline \multicolumn{5}{|l|}{ Tumor location } \\
\hline Upper & 49 & $4.0 \%$ & 12 & $3.3 \%$ \\
\hline Middle & 892 & $72.6 \%$ & 258 & $71.9 \%$ \\
\hline Lower & 287 & $23.4 \%$ & 89 & $24.8 \%$ \\
\hline \multicolumn{5}{|c|}{ Depth of tumor invasion } \\
\hline pT1 & 106 & $8.6 \%$ & 27 & $7.5 \%$ \\
\hline pT2 & 301 & $24.5 \%$ & 87 & $24.2 \%$ \\
\hline pT3 & 793 & $64.6 \%$ & 230 & $64.1 \%$ \\
\hline pT4 & 28 & $2.3 \%$ & 15 & $4.2 \%$ \\
\hline \multicolumn{5}{|c|}{ Lymph node metastasis } \\
\hline pNO & 502 & $40.9 \%$ & 149 & $41.5 \%$ \\
\hline $\mathrm{pN} 1$ & 502 & $40.9 \%$ & 134 & $37.3 \%$ \\
\hline pN2 & 224 & $18.2 \%$ & 76 & $21.2 \%$ \\
\hline \multicolumn{5}{|l|}{ Differentiation } \\
\hline G1 & 61 & $5 \%$ & 15 & $4.2 \%$ \\
\hline G2 & 693 & $56.4 \%$ & 210 & $58.5 \%$ \\
\hline G3 & 474 & $38.6 \%$ & 134 & $37.3 \%$ \\
\hline \multicolumn{5}{|l|}{ Stage } \\
\hline I & 98 & $8 \%$ & 27 & $7.5 \%$ \\
\hline$\|$ & 506 & $41.2 \%$ & 141 & $39.3 \%$ \\
\hline III & 624 & $50.8 \%$ & 191 & $53.2 \%$ \\
\hline
\end{tabular}


Lv et al. BMC Cancer $\quad$ (2021) 21:1072

Page 6 of 11

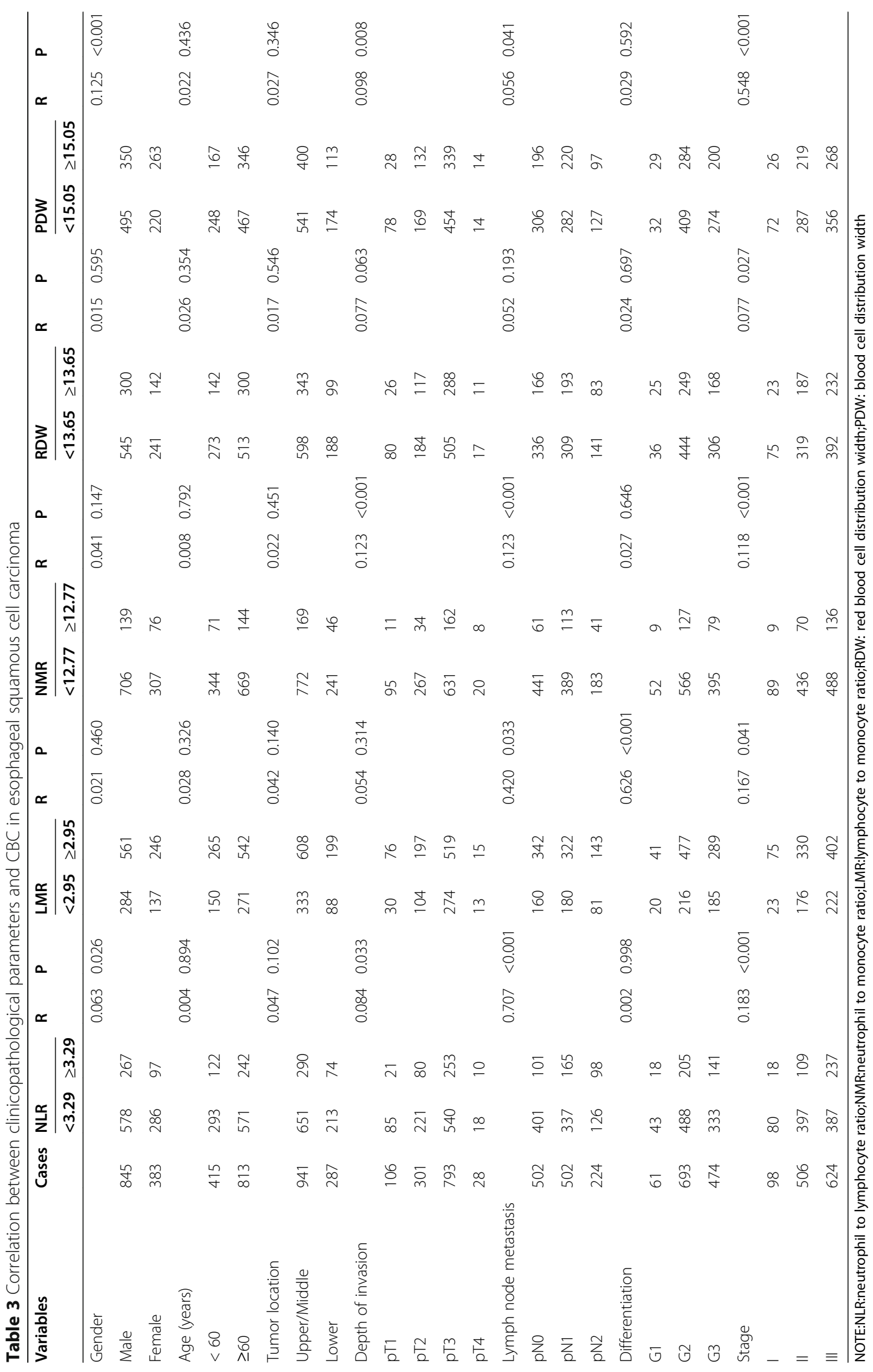


Table 4 Univariate and multivariate Cox proportional hazards regression models in patients with ESCC

\begin{tabular}{|c|c|c|c|c|}
\hline & Univariate analysis HR $(95 \% \mathrm{Cl})$ & $P$-value & Multivariate analysis HR $(95 \% \mathrm{Cl})$ & P-value \\
\hline Age (years) & & $<0.001$ & & $<0.001$ \\
\hline$<60$ years & 1.000 & - & 1.000 & \\
\hline$\geq 60$ years & $1.274(1.112-1.461)$ & - & $1.341(1.169-1.538)$ & \\
\hline Gender & & 0.850 & & - \\
\hline Male & 1.000 & - & - & \\
\hline Female & $0.987(0.861-1.131)$ & - & - & \\
\hline Tumor location & & 0.140 & & - \\
\hline Upper/Middle & 1.000 & - & - & \\
\hline Lower & $1.117(0.964-1.294)$ & - & - & \\
\hline Depth of invasion & & $<0.001$ & & $<0.001$ \\
\hline pT1 & 1.000 & - & 1.000 & - \\
\hline pT2 & $1.402(1.051-1.869)$ & 0.022 & $1.085(0.796-1.479)$ & 0.606 \\
\hline pT3 & $2.404(1.845-3.133)$ & $<0.001$ & $1.557(1.137-2.131)$ & 0.006 \\
\hline pT4 & $3.437(2.168-4.451)$ & $<0.001$ & $2.721(1.527-4.848)$ & 0.001 \\
\hline Lymph node metastasis & & $<0.001$ & & 0.016 \\
\hline pNO & 1.000 & - & 1.000 & - \\
\hline $\mathrm{pN1}$ & $1.725(1.493-1.994)$ & $<0.001$ & $1.354(1.066-1.718)$ & 0.013 \\
\hline pN2 & $2.241(1.881-2.670)$ & $<0.001$ & $1.530(1.142-2.048)$ & 0.004 \\
\hline Stage & & $<0.001$ & & 0.036 \\
\hline । & 1.000 & - & 1.000 & - \\
\hline$\|$ & $1.973(1.456-2.674)$ & $<0.001$ & $1.432(1.007-2.035)$ & 0.046 \\
\hline III & 3.657 (2.712-4.933) & $<0.001$ & 1.705 (1.078-2.696) & 0.023 \\
\hline Differentiation & & 0.122 & & 0.046 \\
\hline G1 & 1.000 & - & & - \\
\hline G2 & $1.411(1.105-1.961)$ & 0.040 & - & - \\
\hline G3 & 1.374 (0.984-1.918) & 0.062 & - & - \\
\hline NLR & & $<0.001$ & & $<0.001$ \\
\hline$<3.29$ & 1.000 & & 1.000 & - \\
\hline$\geq 3.29$ & $1.859(1.678-2.060)$ & & $1.578(1.373-1.814)$ & - \\
\hline NMR & & 0.202 & & - \\
\hline$<12.77$ & 1.000 & & & - \\
\hline$\geq 12.77$ & $2.010(1.716-2.355)$ & & - & - \\
\hline LMR & & $<0.001$ & & 0.001 \\
\hline$\geq 2.95$ & 1.000 & - & 1.000 & - \\
\hline$<2.95$ & $1.373(1.205-1.566)$ & - & $1.265(1.107-1.445)$ & \\
\hline RDW & & 0.049 & & 0.366 \\
\hline$<15.05 \%$ & 1.000 & & 1.000 & - \\
\hline$\geq 15.05 \%$ & $1.1411 .001-1.301)$ & & $1.063(0.931-1.213)$ & - \\
\hline PDW & & 0.016 & & 0.104 \\
\hline$<13.65 \%$ & 1.000 & - & 1.000 & - \\
\hline$\geq 13.65 \%$ & $1.280(1.048-1.562)$ & - & 1.181 (0.966-1.444) & - \\
\hline
\end{tabular}



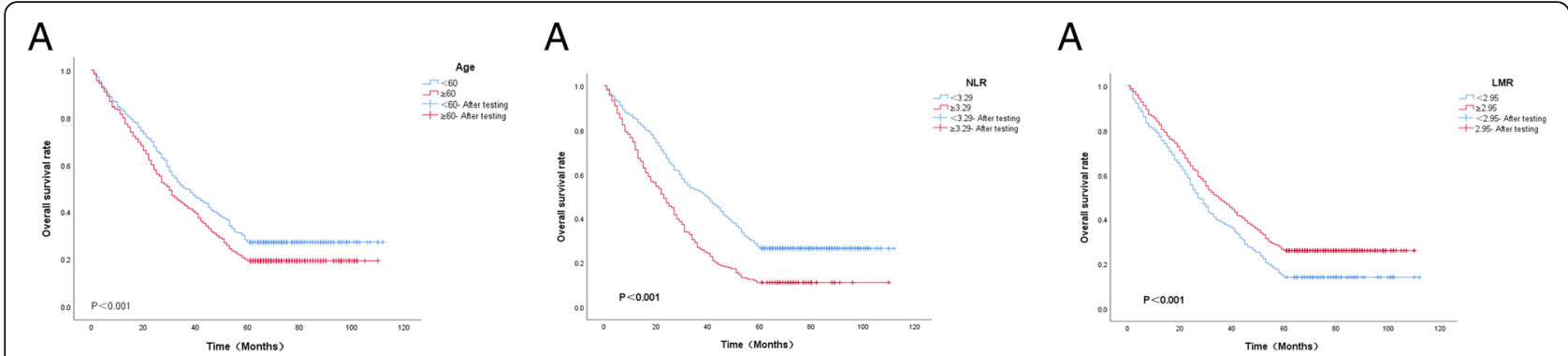

Fig. 2 Kaplan Meier survival curve for the indicators of significance. NOTE: NLR:neutrophil to lymphocyte ratio;LMR:lymphocyte to monocyte ratio

conclusions, Kawakita Y [19] et al. believed that PDW < $12.5 \%$ could predict poor prognosis of esophageal cancer, but in contrast to Song Q [20] et al., he believed that high PDW could predict poor prognosis of esophageal cancer, and in this study, PDW, RDW, and NMR were not independent prognostic factors of preoperative esophageal squamous cell carcinoma $(p>0.05)$, which may be different from the cut-off values of PDW, RDW, and NMR in these studies. The criteria and methods for determining cut-off values were diferent in diferent institutions; a suitable cut-off value cannot be proposed by statistical analysis. This may affect the results and lead to an inevitable potential bias, which may limit the value of NLR, LMR, RDW, PDW in clinical practice and even lead to distinct conclusions, therefore, defining NLR, LMR, RDW, PDW requires a standard, uniform cutoff. Among the pathological parameters, we concluded that pathological stage, depth of invasion, and lymph node metastasis were independent prognostic factors for preoperative esophageal squamous cell carcinoma, and differentiation was not an independent prognostic factor, which may be associated with most of the esophageal cancers being advanced. In this study, we tried to establish a nomogram based on blood routine and pathological parameters to predict the prognosis of ESCC. In the performance validation, its 3year calibration curve indicated that its predictive probability overestimate the actual probability. 5-year calibration curve indicated that its predictive probability was consistent with the actual probability, which may be related to the low overall survival rate of ESCC and needs to be further explored; the decision curve analysis showed the potential of clinical application of the prediction model. This was also verified in the validation cohort. We believe that our model is a simple and easy tool for both physicians and patients to estimate the survival rate of esophageal cancer patients in the postoperative situation.

Several limitations should be acknowledged in the current study. First, the current study is a retrospective study, although it is a large sample, but other potential diseases affecting inflammatory cannot be completely

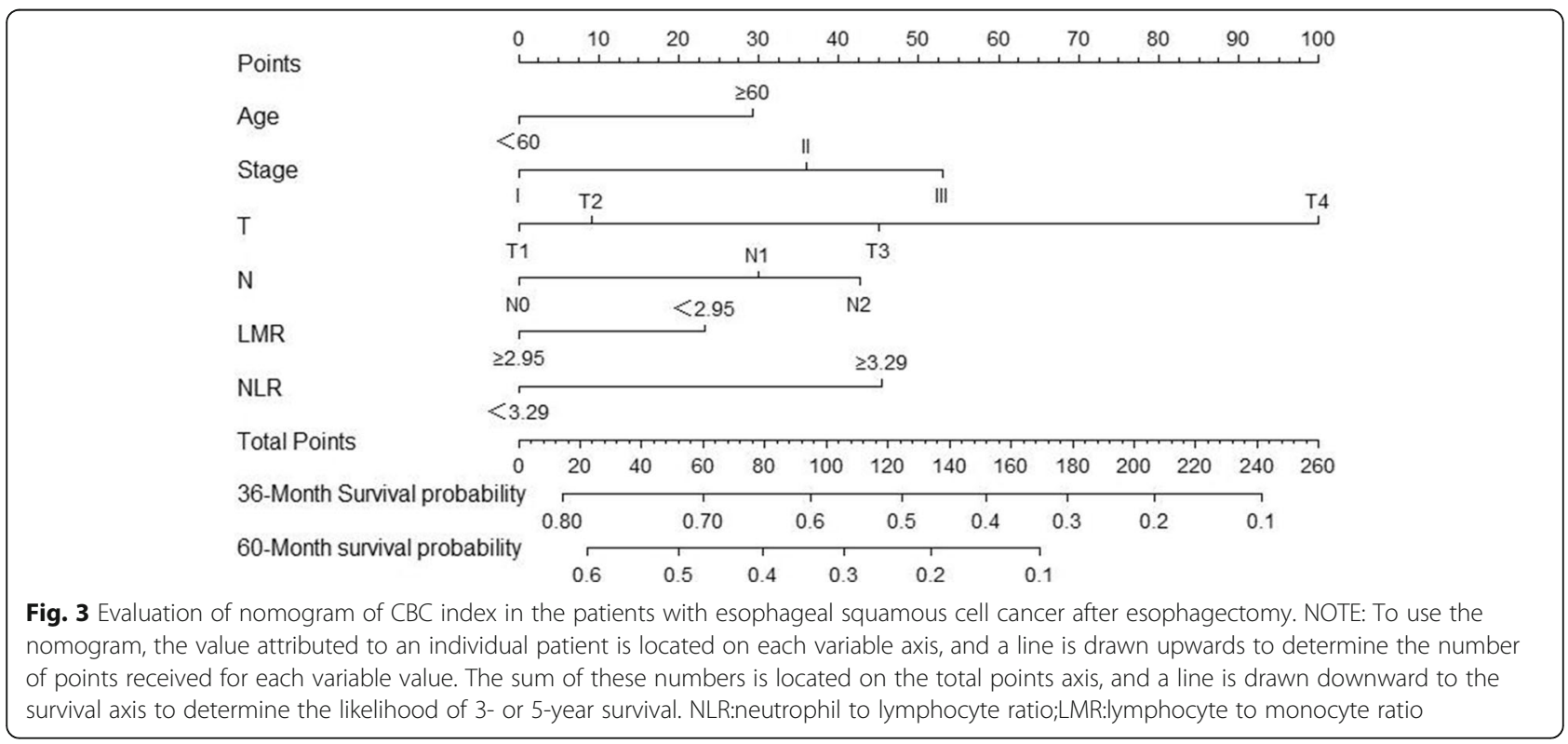




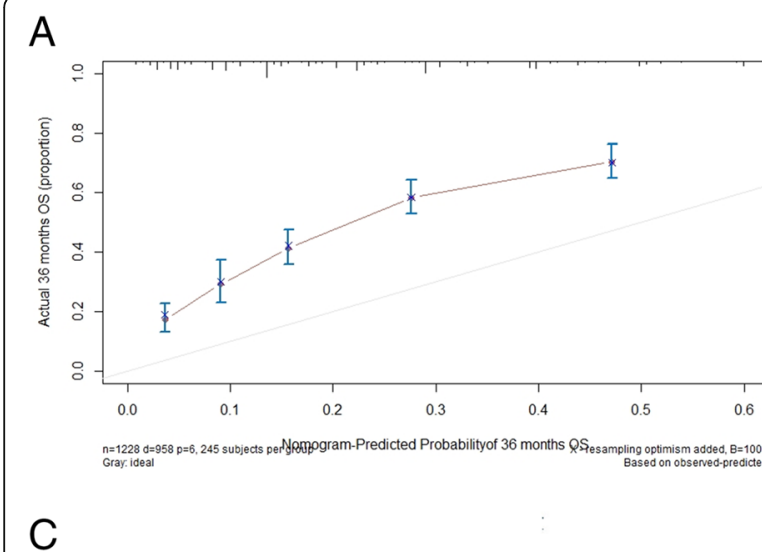

\section{B}

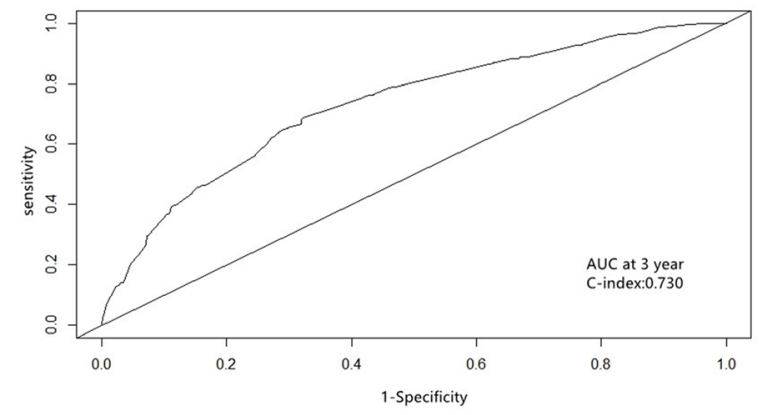

D
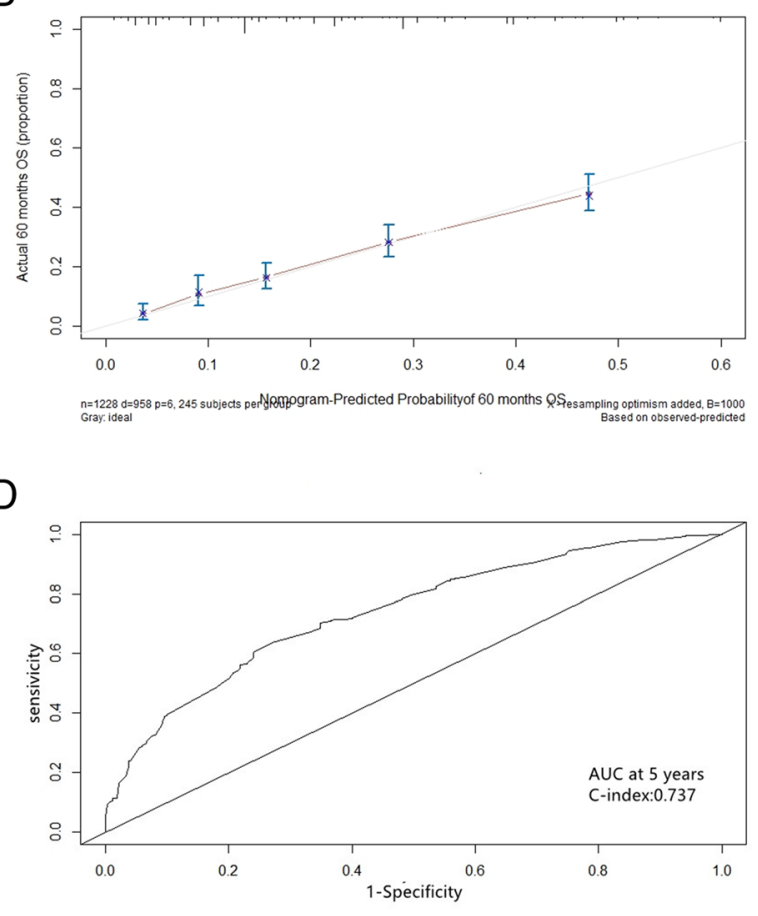

E

F
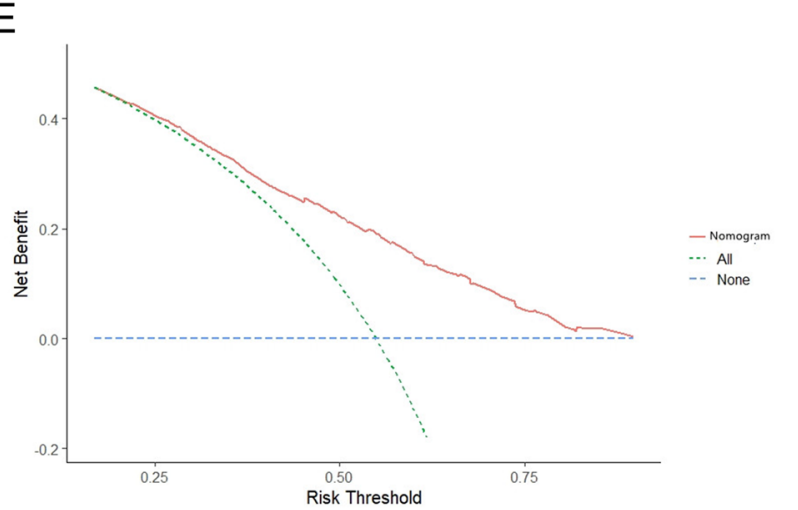

$F$

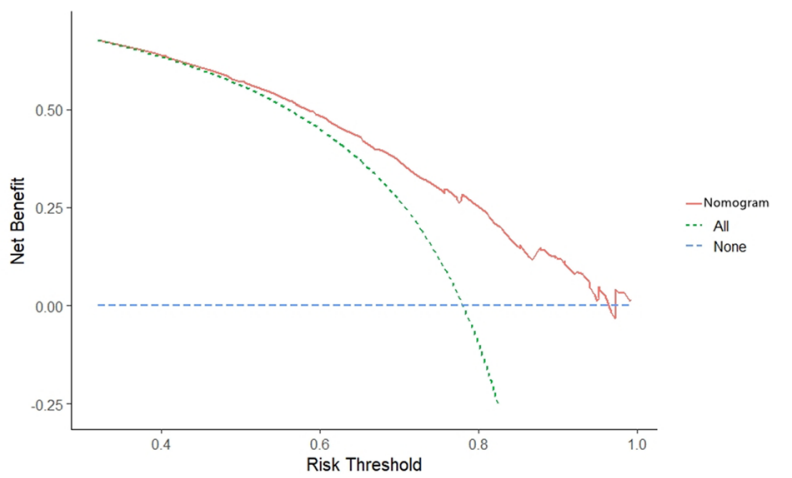

Fig. 4 Performance validation for predicting 3-year and 5-year survival in the training cohort. NOTE: Calibration curve by nomogram, Pathological stage for 3-year (A) and 5-year (B) OS in the training cohort, Time-dependent receiver operating characteristic (ROC) curves by nomogram,

Pathological stage for 3-year (C) and 5-year (D) OS in the training cohort. Decision curve analyses by nomogram, Pathological stage for 3-year (E) and 5-year (F) OS in the training cohort 

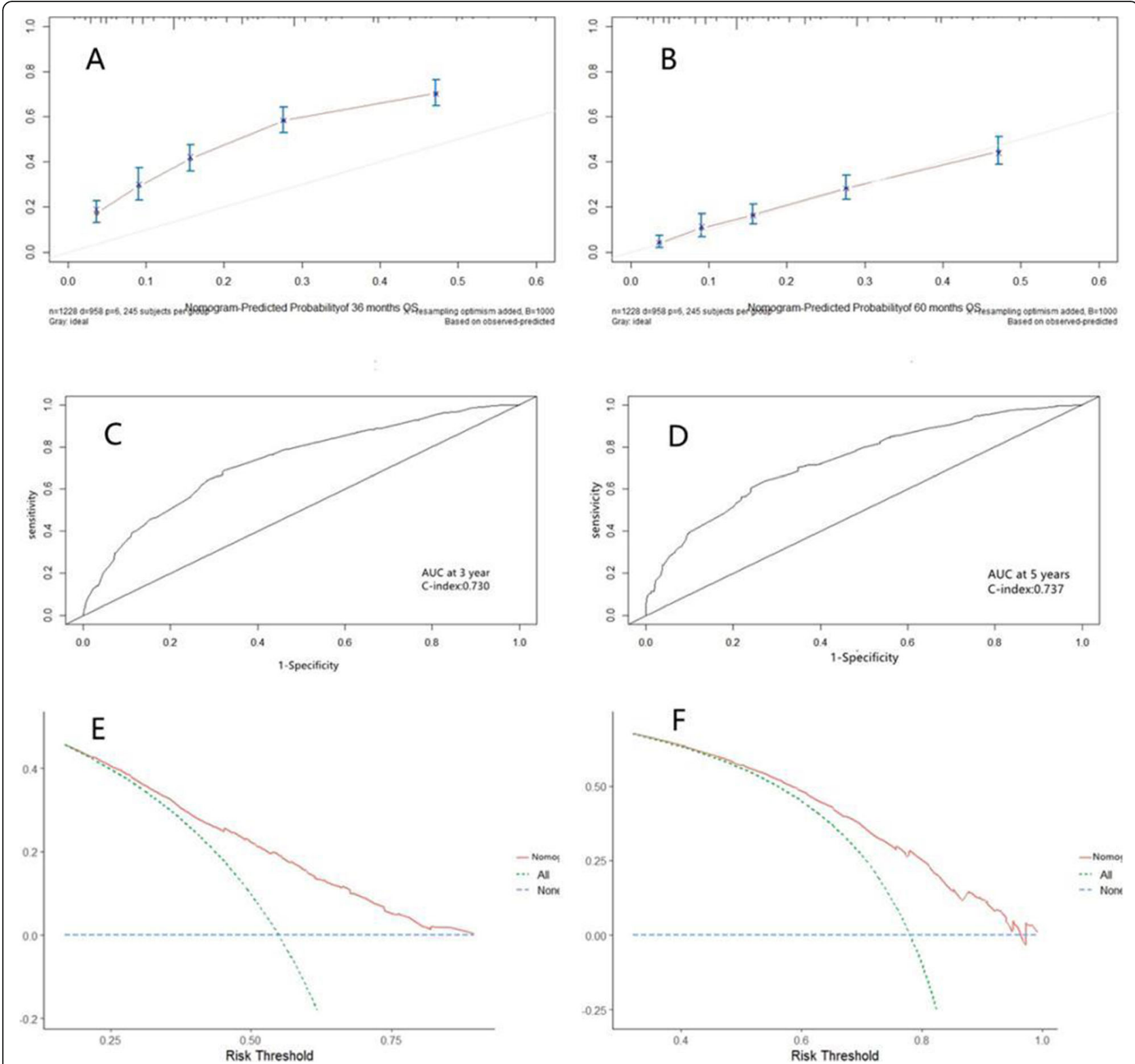

Fig. 5 Performance validation for predicting 3-year and 5-year survival in the validation cohort. NOTE: Calibration curve by nomogram, Pathological stage for 3year (A) and 5-year (B) OS in the validation cohort, Time-dependent receiver operating characteristic (ROC) curves by nomogram, Pathological stage for 3-year (C) and 5-year (D) OS in the validation cohort. Decision curve analyses by nomogram, Pathological stage for 3-year (E) and 5-year (F) OS in the validation cohort 
ruled out. Secondly, excessive uncontrollable factors for laboratory test results of blood cell count-related indicators and excessive variation of indicators, which lead to its limited role in the judgment of clinical prognosis. Therefore, this study aimed at the expression level of peripheral blood cells in patients with esophageal cancer, and explored the relationship between a variety of inflammatory indicators and the prognosis of esophageal cancer, in order to provide new ideas for the prognosis of esophageal cancer. So we also expected to have a more scientific and rigorous prospective, multicenter study to verify our result.

\section{Acknowledgements}

Confirmation of all documents.

\section{Authors'contributions}

(I) Conception and design: Yangchun Feng. (II) Administrative support: Yangchun Feng. (III) Collection and assembly of data: Xiang Lv, Songtao Han, Bin Xu, Yuqin Deng. (IV) Data analysis and interpretation: Xiang LV, Songtao Han, Bin Xu.(V) Manuscript writing: All authors.(VI) Final approval of manuscript: All authors.

\section{Funding}

No funding.

\section{Availability of data and materials}

The datasets generated and/or analysed during the current study are not publicly available due requirements of Cancer hospital Affliated to Xinjiang Medical University, but it are available from the corresponding author on reasonable request

\section{Declarations}

\section{Ethics approval and consent to participate}

The study was approved by Ethics Committee of the Cancer Hospital Affliated to Xinjiang Medical University. The study confirming that informed consent was obtained from all subjects or, if subjects are under 16, from a parent and/or legal guardian.

The study confirm that all methods were carried out in accordance with relevant guidelines and regulations.

\section{Consent for publication}

Consent for publication.

\section{Competing interests}

The author declares that he has no conflict of interests.

\section{Author details}

'Clinical Laboratory Center, Cancer Hospital Affiliated to Xinjiang Medical University, Xinjiang, China. ${ }^{2}$ Clinical Laboratory Center, Hospital of Traditional Chinese Medicine affiliated to Xinjiang Medical University, Xinjiang, China.

${ }^{3}$ West China hospital affiliated to Sichuan University, Sichuan, China.

Received: 1 June 2021 Accepted: 15 September 2021

Published online: 30 September 2021

\section{References}

1. Bray F, Ferlay J, etal. Global cancer statistics 2018: GLOBOCAN estimates of incidence and mortality worldwide for 36 cancers in 185 countries. CA Cancer J Clin; 68 (6): 394-424, https://doi.org/10.3322/caac.21492.

2. Rustgi AK, El-Serag HB. Esophageal carcinoma. N Engl J Med. 2014 Dec 25; 371(26):2499-509. https://doi.org/10.1056/NEJMra1314530.

3. Feng KY, Quan RL. MiR-206 inhibits the migration and invasion of esophageal cancer cells by targeting ARPC3 [J]. J Huazhong Univ Sci Techn: Med Ed. 2019;48(2):172-6.

4. Huiling Y. Study on the application of humanized nursing in the nursing of senile esophageal cancer radiotherapy [J]. Renowned Doctor. 2020;10:222-3.
5. Wan H, Li L. Clinical application of high quality nursing in the whole process of nursing patients with esophageal cancer [J]. J Nursing Clin Pract. 2020;5(22):40.

6. Lin Y, Peng Y, Chen Y, Li S, Huang $X$, Zhang $H$, et al. Association of lymphocyte to monocyte ratio and risk of in-hospital mortality in patients with acute type a aortic dissection [J]. Biomark Med. 2019;13(15):1263-72. https://doi.org/10.2217/bmm-2018-0423.

7. Li K, Xia XF, Su M, Zhang H, Chen WH, Zou CL. Predictive value of lymphocyte-to-monocyte ratio (LMR) and concurrent neutrophil-tolymphocyte ratio (NLR) in patients with oesophageal cancer undergoing chemoradiotherapy [J]. BMC Cancer. 2019;19(1):1004. https://doi.org/10.11 86/s12885-019-6157-4.

8. Liang H, Fan JH, Qiao YL. Epidemiology, etiology, and prevention of esophageal squamous cell carcinoma in China. Cancer Biol Med. 2017 Feb; 14(1):33-41. https://doi.org/10.20892/j.issn.2095-3941.2016.0093.

9. Xie DD, Chen YH, Xu S, Zhang C, Wang DM, Wang H, et al. Low vitamin D status is associated with inflammation in patients with prostate cancer. Oncotarget. 2017 Mar 28;8(13):22076-85. https://doi.org/10.18632/oncota rget.16195,

10. Lussana F, Rambaldi A. Inflammation and myeloproliferative neoplasms. J Autoimmun. 2017 Dec;85:58-63. https://doi.org/10.1016/j.jaut.2017.06.010.

11. Zhang A, Ning L, Han J, et al. Neutrophil-to-lymphocyte ratio as a potential biomarker of Neovascular Glaucoma [J]. Ocul Immunol Inflamm. 2019;10:1-8.

12. Jinjing $G$, Linhui $H$, et al. The value of red blood cell distribution width in the staging prognostic of multiple myeloma [J]. J Modern Lab Med. 2017; 32(3):34-6 39

13. Shao $Y$, Ning $Z$, et al. Prognostic nomogram integrated systemic inflammation score for patients with esophageal squamous cell carcinoma undergoing radical esophagectomy. Sci Rep. 2015;5(1):18811. https://doi. org/10.1038/srep18811.

14. Arigami $T$, Okumura $H$, Matsumoto $M, U$ Chikado $Y$, Uenosono $Y$, Kita $Y$, et al. Analysis of the fibrinogen and neutrophil-lymphocyte ratio in esophageal squamous cell carcinoma: a promising blood marker of tumor progression and prognosis. Medicine (Baltimore). 2015;94(42):e1702. https://doi.org/10.1 097/MD.0000000000001702.

15. Gao GD, Sun B, Wang XB, Wang SM. Neutrophil to lymphocyte ratio as prognostic indicator for patients with esophageal squamous cell cancer. Int J Biol Markers. 2017 Oct 31;32(4):e409-14. https://doi.org/10.5301/ijbm.5000294.

16. Huang Y, Feng JF. Low preoperative lymphocyte to monocyte ratio predicts poor cancer-specific survival in patients with esophageal squamous cell carcinoma. Onco Targets Ther. 2015 Jan 8;8:137-45. https://doi.org/10.2147/ OTT.S73794.

17. Hu G, Liu G. Ma JY, etal Lymphocyte-to-monocyte ratio in esophageal squamous cell carcinoma prognosis. Clin Chim Acta. 2018;486:44-8. https:// doi.org/10.1016/j.cca.2018.07.029.

18. Li KJ, Xia XF, Su M, et al. Predictive value of lymphocyte-to-monocyte ratio (LMR) and neutrophil-to-lymphocyte ratio (NLR) in patients with oesophageal cancer undergoing concurrent chemoradiotherapy. BMC Cancer. 2019;19(1):1004.

19. Kawakita Y, Motoyama S. Etal. Prognostic significance of combined platelet distribution width and C-reactive protein score in esophageal Cancer. Anticancer Res. 2020 Oct;40(10):5715-25. https://doi.org/10.21873/antica nres. 14586 .

20. Song Q, Wu JZ, Wang S, Chen WH. Elevated preoperative platelet distribution width predicts poor prognosis in esophageal squamous cell carcinoma. Sci Rep. 2019 Oct 23;9(1):15234. https://doi.org/10.1038/s41598019-51675-y.

\section{Publisher's Note}

Springer Nature remains neutral with regard to jurisdictional claims in published maps and institutional affiliations. 